\title{
SOME APPROXIMATION PROPERTIES OF HEXAGONAL FOURIER SERIES
}

\begin{abstract}
Ali Guven
Abstract. L. Leindler, A. Meir and V. Totik considered the $\varphi$-norm on $C_{2 \pi}$ (the space $2 \pi$ periodic continuous functions) and estimated the deviation $\left\|A_{n}(f)-f\right\|_{\varphi}$ in terms of the modulus of continuity of $f \in C_{2 \pi}$, where $\left(A_{n}\right)$ is a sequence of convolution operators from $C_{2 \pi}$ into itself and $\varphi$ is an increasing function on $(0, \infty)$ (Acta Math. Hung. 45 (1985), 441-443). In the present paper, an analogue of the theorem of Leindler, Meir and Totik is proved for functions periodic with respect to the hexagon lattice. Also, this theorem is applied to obtain estimates for approximation by partial sums of hexagonal Fourier series in Hölder and generalized Hölder norms.
\end{abstract}

Mathematics subject classification (2010): 41A25, 42A10.

Keywords and phrases: Hexagonal Fourier series, modulus of continuity, $\varphi$-norm.

\section{REFERENCES}

[1] J. Bustamante And M. A. Jimenez, Trends in Hölder approximation, In: Approximation, optimization and mathematical economics (M. Lassonde ed.), Springer (2001).

[2] R. A. DeVore and G. G. Lorentz, Constructive approximation, Springer-Verlag, Berlin, 1993.

[3] B. FugLede, Commuting self-adjoint partial differential operators and a group theoretic problem, J. Functional Analysis 16 (1974), 101-121.

[4] A. Guven, Approximation by means of hexagonal Fourier series in Hölder norms, J. Classical Anal. 1 (2012), 43-52.

[5] A. Guven, Approximation by $(C, 1)$ and Abel-Poisson means of Fourier series on hexagonal domains, Math. Inequal. Appl. 16 (2013), 175-191.

[6] A. Guven, Approximation properties of hexagonal Fourier series in the generalized Hölder metric, Comput. Methods Funct. Theory 13 (2013), 509-531.

[7] A. Guven, Approximation of continuous functions by de la Vallèe-Poussin of Fourier series on hexagonal domains, Jaen J. Approx. 5 (2013), 61-80.

[8] A. Guven, An analogue of Leindler's theorem for hexagonal Fourier series, Analysis 34 (2014), 283-297.

[9] L. LeIndLER, Generalizations of Prössdorf's theorems, Studia Sci. Math. Hung. 14 (1979), 431-439.

[10] L. Leindler, A. Meir And V. TotiK, On approximation of continuous functions in Lipschitz. norms, Acta Math. Hung. 45 (1985), 441-443.

[11] H. LI, J. SUN AND Y. XU, Discrete Fourier analysis, cubature and interpolation on a hexagon and a triangle, SIAM J. Numer. Anal. 46 (2008), 1653-1681.

[12] A. N. PODKORYTOV, Asymptotics of the Dirichlet kernel of Fourier sums with respect to a polygon, J. Sov. Math. 42 (1988), 1640-1646.

[13] S. PRÖSSDORF, Zur konvergenz der Fourierreihen hölderstetiger funktionen, Math. Nachr. 69 (1975), $7-14$.

[14] J. Sun, Multivariate Fourier series over a class of non tensor-product partition domains, J. Comput. Math. 21 (2003), 53-62.

[15] A. F. Timan, Theory of approximation of functions of a real variable, Pergamon Press, New York, 1963. 
[16] Y. XU, Fourier series and approximation on hexagonal and triangular domains, Constr. Approx. 31 (2010), 115-138.

[17] A. Zygmund, Trigonometric series, Cambridge Univ. Press, New York, 1959. 